\title{
Von Li Bai bis Mao Zedong \\ Chinesische Dichterhelden in der deutschen Lyrik des 20. Jahrhunderts
}

\section{Sara Landa}

\section{Einleitung}

1938 schrieb Bertolt Brecht im dänischen Exil ein Gedicht mit dem Titel Besuch bei den verbannten Dichtern, das folgendermaßen beginnt:

Als er im Traum die Hütte betrat der verbannten

Dichter, die neben der Hütte gelegen ist

Wo die verbannten Lehrer wohnen (er hörte von dort

Streit und Gelächter), kam ihm zum Eingang

Ovid entgegen und sagte ihm halblaut:

„Besser, du setzt dich noch nicht. Du bist noch nicht gestorben. Wer weiß da

$\mathrm{Ob}$ du nicht doch noch zurückkehrst? Und ohne daß andres sich ändert

Als du selber." Doch, Trost in den Augen

Näherte Po Chü-i sich und sagte lächelnd: „Die Strenge

Hat sich jeder verdient, der nur einmal das Unrecht benannte.“

Und sein Freund Tu-Fu sagte still: „Du verstehst, die Verbannung

Ist nicht der Ort, wo der Hochmut verlernt wird." $[\ldots]^{1}$

Die Reihe der Dichter wird fortgesetzt mit François Villon, Voltaire, Shakespeare, Heine und Euripides, die dem lyrischen Ich wohlwollend Ratschläge erteilen. Dann wird die entspannte Atmosphäre jäh unterbrochen, als „aus der dunkelsten Ecke“2 eine Frage kommt:

$[\ldots]$ „Du, wissen sie auch

Deine Verse auswendig? Und die sie wissen

Werden sie der Verfolgung entrinnen?" „Das

Sind die Vergessenen“, sagte der Dante leise

„Ihnen wurden nicht nur die Körper, auch die Werke vernichtet.“

Das Gelächter brach ab. Keiner wagte hinüberzublicken. Der Ankömmling

War erblaßt. ${ }^{3}$

Das Gedicht suggeriert eine solidarische, welt- und zeitumspannende Gemeinschaft der Dichter gegenüber der Herrschaft, stellt aber auch deren Bedrohtheit

1 Bertolt Brecht: Werke. Große kommentierte Berliner und Frankfurter Ausgabe, hg. von Werner Hecht u. a., Bd. 12: Gedichte 2: Sammlungen 1938-1956, Berlin/Frankfurt am Main 1988, S. 35.

2 Ebd.

3 Ebd., S. 35-36. 
heraus. ${ }^{4}$ Dass Brecht in diesem Text unter den Dichtervorbildern auch zwei chinesische Schriftsteller, nämlich die Tang-Dichter $\mathrm{Du} F u$ (杜甫, auch Tu-Fu, Thu-Fu) ${ }^{5}$ und Bai Juyi (白居易, auch Bo Juyi, Po Chü-I, Pe-Lo-Thien) nennt, ist letztendlich nicht so überraschend. Tatsächlich dienten fernöstliche Dichter vor allem seit Beginn des 20. Jahrhunderts zahlreichen deutschen Autoren als Orientierungsfiguren. Das Interesse an der chinesischen Lyrik, das im frühen 20. Jahrhundert kulminierte, war zentral mitbestimmt von der kreativen Auseinandersetzung mit den Biographien ihrer Erschaffer. Gerade in Zeiten literarischer, politischer und persönlicher Umbrüche, in denen die deutschen Dichter sich mit ihrer eigenen Rolle auseinandersetzten, wurden die Biographien der chinesischen Vorbilder aufgegriffen, aber auch in vieler Hinsicht verändert, weitergesponnen und einem gewissen Heroisierungsprozess unterworfen. Dabei wurden diese Dichterbilder sowohl durch Dichtergedichte ${ }^{6}$ gestaltet als auch in Transformationen ${ }^{7}$ chinesischer Dichtung, die autobiographische Lesarten nahelegen und mit Vor- und Nachworten versehen sind. Das Heldenbild konstituiert sich so im Zusammenspiel von lyrischer (Auto-)Biographik und Paratext. Legitimiert wird die Heroisierung durch das Zusammentreffen ästhetischer Kategorien mit moralischen bzw. soziopolitischen Leistungen. Somit wird auch danach zu fragen sein, wie das Konzept des Kulturheros als eines Kulturbringers, herausragenden Schöpfers, ${ }^{8}$ mit anderen Heldenvorstellungen interferiert und welche Rolle dies für die Aushandlung des dichterischen Selbstverständnisses im 20. Jahrhundert spielte.

4 Vgl. Ulrich Kittstein: Das lyrische Werk Bertolt Brechts, Stuttgart/Weimar 2012, S. 219; Werner Frick: „....und er hörte von dort Streit und Gelächter“: Der Lyriker Bertolt Brecht im ,Club der toten Dichter', in: Helmut Koopmann (Hg.): Brechts Lyrik - Neue Deutungen, Würzburg 1999, S. 75-99, hier S. 95-97.

5 Die deutschen Schreibungen der chinesischen Namen variieren stark. Der besseren Zuordnung halber werden jeweils die heute übliche Pinyin-Umschrift, der chinesische Name im Original sowie einige gängige Umschriftvarianten aufgeführt.

6 Zum Terminus des Dichtergedichts als Text, der ,über den Poeten im allgemeinen, über andere Dichter oder über die eigene Dichterexistenz reflektier[t]", siehe Heinz Schlaffer: Das Dichtergedicht im 19. Jahrhundert. Topos und Ideologie, in: Jahrbuch der deutschen Schillergesellschaft 10, 1966, S. 297-335, hier S. 297. Schlaffer zeigt auch auf, wie ausgeprägt die idealistischen Stilisierungen der Dichterfiguren in den Dichtergedichten oft sind.

7 Ich verwende den Begriff der Transformation in Anlehnung an Michael Schreiber (Übersetzung und Bearbeitung. Zur Differenzierung und Abgrenzung des Übersetzungsbegriffs, Tübingen 1993, S. 7) als Überbegriff für Übersetzungen und Bearbeitungen. Die Grenze zwischen diesen Aneignungsformen ist im Falle der vielfach indirekten Annäherung an die chinesische Lyrik oft schwer zu ziehen und soll nicht Thema dieses Aufsatzes sein.

8 Zum Konzept des Kulturheros vgl. insbesondere Zaal Andronikashvili u. a.: Einleitung, in: dies. (Hg.): Kulturheros. Genealogien. Konstellationen. Praktiken (LiteraturForschung 28), Berlin 2017, S. 9-38, v. a. S. 12-13. Überschneidungen zwischen der literarischen und der politischen Ebene sind auch bei zahlreichen anderen Dichterhelden gegeben (vgl. z. B. Christoph Schmälzle: Dichterkult und nationales Heil. Friedrich Schiller als Garant „deutscher Größe“, in: Andronikashvili u. a. [Hg.]: Kulturheros, S. 242-281), im Falle der China-Rezeption aber besonders ausgeprägt. 
Im Folgenden möchte ich daher anhand einzelner Fallbeispiele zeigen, welche Formen die Stilisierung chinesischer Dichterhelden im 20. Jahrhundert annehmen konnte.

\section{Genie und Vagant: Li Bai in der deutschen Lyrik}

Zu keiner Zeit war die Auseinandersetzung mit den chinesischen Dichtern und der chinesischen Dichtung so intensiv wie zu Beginn des 20. Jahrhunderts, als China entweder als veränderungsunfähig oder revolutionäres Vorbild, als Ursprungsort philosophischer Heilsbringer oder aber als militärische Bedrohung galt. ${ }^{9}$ Vor allem Li Bai (李白, auch Li Bo, Li Tai-po, Li Tai-pe), der u. a. für seine lyrisch inszenierten Trunkenheitszustände und spielerischen Selbsterhöhungen bekannte Tang-Dichter, zog die deutschen Dichter in seinen Bann. Li Bai galt den Dichtern um die Jahrhundertwende als „ewig trunkener, ewig heiliger Wanderer“ und „erlauchter Vagabund", ${ }^{10}$ so Klabund, der ihn immer wieder in die Nähe François Villons rückt. ${ }^{11}$

Die Faszinationskraft Li Bais, aber auch eine gewisse kritische, dialogische Auseinandersetzung mit dem chinesischen Dichter werden beispielsweise im Werk Richard Dehmels offensichtlich. So integriert Dehmel in seinen Band Aber die Liebe einen kleinen Zyklus an Trinkliedern, drei nach Li Bai sowie ein eigenes, ${ }^{12}$ und stellt sich so direkt in die Tradition des Chinesen. Einem der Li Bai-Texte wiederum schreibt er durch Hinzufügung einer eigenen Strophe einen Dialog mit dem Prätext besonders deutlich ein. Es handelt sich um Li Bais berühmtes 春日醉 起言志 (Worte beim betrunkenen Erwachen an einem Früblingstag):

Richard Dehmel: Frühlingsrausch

Wenn das Leben Traum ist, wie sie meinen, wozu dann ihre nüchterne Plage!

Ich, ich berausche mich alle Tage;

und wenn ich Nachts nichts mehr vertrage,

5 leg ich mich schlafen auf den Pflastersteinen!

Morgens erwach ich sehr bewußt;

ein Vogel zwitschert zwischen blühenden Reben.

Ich frage ihn, in welcher Zeit wir leben.

Er sagt mir: in der Zeit der blühenden Reben!

10 das ist die Zeit, in der die Frühlingslust

die Vögel zwitschern lehrt und leben, leben!

9 Vgl. Andreas Stehen (Verf.) / Mechthild Leutner (Hg.): Deutsch-chinesische Beziehungen 1911-1927. Vom Kolonialismus zur „Gleichberechtigung“. Eine Quellensammlung, Berlin 2006, S. 495.

10 Klabund [Alfred Henschke]: Werke in acht Bänden, hg. von Christian v. Zimmermann, Bd. 7: Übersetzungen und Nachdichtungen, Heidelberg 2001, S. 65.

11 Vgl. bspw. die Li Bai- und Villon-Gedichte in: ebd., S. 46 sowie Bd. 4.1, S. 133.

12 Vgl. Richard Dehmel: Aber die Liebe. Zwei Folgen Gedichte, Berlin 1912, S. 62-66. 
Ich bin erschüttert; ich raff mich auf wie toll, wütende Seufzer pressen mir die Kehle. Und wieder gieß ich mir den Becher voll, bis in die Nacht, und pfeif auf meine Fehle.

Wenn dann mein Mund ausruht, ruht auch mein Groll, ruht alles, was ich will und kann und soll, ruht rings die Welt - o ruhte auch die Seele!

Wer aber kann mit Wein den Gram verjagen? wer kann das Meer mit einem Schluck verschlingen?

Der Mensch, in diesen Lebensrausch verschlagen, in dem sich Sehnsucht und Erfüllung jagen, kann nichts tun als in einen Nachen springen, mit flatterndem Haar im Wind die Mütze schwingen

25 und, während ihn die Elemente tragen, sich ihrer Willkür stolz zum Opfer bringen! $!^{13}$

Das Ich des Gedichts, mit seinem Autor assoziiert, erwacht aus dem Rausch und reflektiert über die Illusionshaftigkeit und Nichtigkeit des Daseins, um sich dann wieder im Rausch von allem Gefühl für die Welt zu befreien. Dehmel jedoch stellt diese Handlung durch die vierte Strophe, zu der es kein Pendant im Chinesischen oder in früheren europäischen Übertragungen gibt, ${ }^{14}$ explizit in Frage: „Wer aber kann mit Wein den Gram verjagen? / wer kann das Meer mit einem Schluck verschlingen?" Man kann die Fragen lesen als die des Ichs, das sich aus seiner eigenen Melancholie herausreißt, oder aber als Einwürfe einer weiteren Instanz - potentiell assoziiert mit dem deutschen Dichter -, die mit der Frage und Weisung dem Ich widerspricht. In jedem Fall formuliert Dehmel eine Art vitalistische Antwort auf Li Bai, eine Selbstbehauptung des Ich gerade im Exzess und im unvermeidlichen Untergang. Die neugewonnene Vitalität spiegelt sich auch in der vorwärtsdrängenden Dynamik des jambischen Fünfhebers, der im Gegensatz zu den ersten drei Strophen, die mehr Unregelmäßigkeiten aufweisen, fast ausschließlich durchgehalten wird. ${ }^{15}$ Dehmel tritt in einen Dialog mit Li Bai, formuliert eine Antwort auf ihn aus der eigenen Zeit heraus und modifiziert damit das Dichter- und Lebensideal. Das Ideal des lässigen, Konventionen unterlaufenden Dicherrebells wird mit einer Vision desillusionierten, aber tatkräftigen Heldentums konfrontiert, ohne dass eindeutig klar wird, ob dieses Li Bai zugeschrieben oder ihm entgegengesetzt wird.

13 Ebd., S. 64-65.

14 Vgl. Dehmels Vorlage: Hans Heilmann: Chinesische Lyrik vom 12. Jahrhundert v. Chr. bis zur Gegenwart (Die Fruchtschale 1), München/Leipzig 1905, S. 28-29. Ebenso wenig findet sich eine vergleichbare Strophe in irgendeiner der mir bekannten Versionen des damals sehr populären Gedichts.

15 Die einzige Abweichung findet sich in der zusätzlichen Senkung in „flatterndem“, die aber der Dynamik kaum einen Abbruch tut. 


\section{Traditionelle Dichterhelden im Kampf gegen Armut und Unrecht: Du Fu und Bai Juyi}

Li Bai sollte noch lange Zeit populär bleiben, daneben trat aber ein weiterer Rezeptionsstrang. Klabund und vor allem Albert Ehrenstein lenkten seit dem Ersten Weltkrieg und im Kontext der Xinhai-Revolution die Aufmerksamkeit auch auf eine andere Gruppe chinesischer Gedichte, nämlich solche Texte, die Krieg und soziales Elend spiegeln. Du Fu, der neben Li Bai bekannteste, bisher stark unterrepräsentierte Tang-Dichter, und vor allem Bai Juyi, der dank der Übertragungen der Sinologen August Pfizmair, Leopold Woitsch und Arthur Waley zugänglich war, galten nun als prototypische Kämpfer für Gerechtigkeit. Du Fu taucht schon in den chinesischen Sammlungen der Expressionisten vermehrt auf, wurde aber später beispielsweise auch von Klara Blum (Zhu Bailan 朱白兰), einer jüdischen sozialistischen Dichterin aus der Bukowina, die nach China emigrierte, im Kontrast zu Li Bai porträtiert. In Zwei Dichter (aus dem Band Wir entscheiden alles von 1941) lässt Blum die Großmeister sich gegenseitig schreiben und dabei Fremd- und Eigenporträts gestalten. Du Fu als dem Vertreter einer engagierten Ästhetik bleibt das letzte Wort vorbehalten:

\footnotetext{
Mein Freund, bleib Li Tai-Po, ich bleib Thu-Fu.

Denn hast du Deine - ich hab meine Freuden.

Daß jede Macht an meinem Stolz zerstiebt,

Berauscht mich mehr, als Dich der Duft der Weine,

$[\ldots]$

So tret ich täglich denn zum Zweikampf an:

Ich und das bittre Unrecht - wer ist stärker?

Ist diese schwere Art auch nicht Dein Fall,

So zürne mir doch nicht und nicht ergrimme,

Daß deinem Wohllaut, Chinas Nachtigall,

Ich Antwort gab mit einer rauhen Stimme. ${ }^{16}$
}

Noch populärer wird Bai Juyi. Ehrenstein preist ihn im Vorwort zu China klagt als „Ankläger, der den Übermut und die Verschwendung der Mandarine und Fürsten geißelte, den Schrei der leidenden und hungernden Massen ausstieß ${ }^{\text {“ }}{ }^{17}$ Als solcher figuriert er dann auch, wie bereits angedeutet, bei Bertolt Brecht. Brecht schreibt, wie unter anderem auch Yuan Tan herausgestellt hat, ${ }^{18}$ für seine Anmerkungen zu seinem Zyklus Sechs chinesische Gedichte (1938) Passagen aus einer Anthologie des

16 Klara Blum: Kommentierte Auswahledition, hg. von Zhidong Yang, Wien u. a. 2001, S. 326. Vgl. zu dem Gedicht weiter Zhidong Yang: Klara Blum - Zhu Bailan (1904-1971). Leben und Werk einer österreichisch-chinesischen Schriftstellerin, Frankfurt a. M. u. a. 1996, S. 134-135.

17 Albert Ehrenstein: China klagt: Nachdichtungen revolutionärer chinesischer Lyrik aus drei Jahrtausenden (Malik-Bücherei 8), Königstein im Taunus 1981, S. 5.

18 Vgl. Yuan Tan: Under the Chinese Mask: Brecht's „Six Chinese Poems“ Revisited / Unter der chinesischen Maske: Neue Studien zu Brechts „Sechs chinesischen Gedichten“, in: The Brecht Yearbook 36, 2011, S. 150-163, hier S. 152-153. 
britischen Gelehrten Arthur Waley so um, dass Bai Juyi von einem Dichter für alle zu einem Dichter der unteren Schichten (und damit chinesischen Gegenpart Brechts) wird: Heißt es bei Waley noch: „His poems were ,on the mouths of kings, princes, concubines, ladies, plough-boys, and grooms “" ${ }^{19}$ streicht Brecht die oberen Schichten: „Seine Lieder waren ,im Mund von Bauern und Pferdeknechten'. “20 In einigen Gedichtübertragungen folgt Brecht seiner Vorlage auch relativ eng, in anderen wiederum schreibt er den Text komplett um, stilisiert Bai Juyi von einem Autor, der Mitleid mit den Armen empfindet, zum Proto-Revolutionär und Vorläufer der eigenen Poetik um. So macht er in einem Gedicht, das schon in der englischen Fassung letztlich nur einen kleinen Ausschnitt des Originals wiedergibt, ${ }^{21}$ das lyrische Ich vom mitfühlenden Vertreter der Oberschichten zu einem Kritiker, der die Macht explizit herausfordert:

Arthur Waley: The Big Rug

That so many of the poor should suffer from cold what can we do to prevent?

To bring warmth to a single body is not much use.

I wish I had a big rug ten thousand feet long.

Which at one time could cover up every inch of the City. ${ }^{22}$

Bertolt Brecht: Die Decke

Der Gouverneur, von mir befragt,

Was, den Frierenden in unsrer Stadt zu helfen, nötig sei

Antwortete: Eine zehntausend Fuß lange Decke

Welche die ganzen Vorstädte einfach zudeckt. ${ }^{23}$

Kontrastiert der chinesische Text noch die Freude über die eigene neue Kleidung mit dem schlechten Gewissen wegen dem Leid der Armen und erhält so selbstkritische Züge, reduziert Waley den Text auf das Moment des Empathischen. Durch das naiv-hyperbolische Bild der riesigen Decke und den Irrealis kippt die Klage ins Sentimentalische. Brecht wiederum teilt die Rollen auf und schafft ein kritisch fragendes Ich, das den Vertreter der Macht konfrontiert. Die Antwort des Gouverneurs lässt sich als Ausdruck von Verlegenheit und Überforderung lesen oder aber als Zynismus, indem suggeriert wird, die Regierung wolle die Probleme vertuschen. ${ }^{24}$ Das Ich als kritischer Beobachter ist dabei sicherlich mit dem Autor

19 Arthur Waley (Übers.): One Hundred \& Seventy Chinese Poems, London 1919, S. 112.

20 Brecht: Werke (Anm. 1), Bd. 22, S. 454.

21 Es handelt sich um 新制绫袄成感而有咏 (Lied zu den Gefüblen bei der Fertigstellung einer neuen Seidenjacke). Ein ähnliches Gedicht trägt den Titel 新制布表 (Der neue Pelzmantel). Aufgrund der starken Kürzungen Waleys ist das Original nicht eindeutig zuordbar, die Erwähnung der Stadt spricht aber für den ersteren Text (vgl. Shi Jie 史节: Chinesische kulturelle Elemente in Brechts Gedichten 布莱希特诗歌作品中的中国文化元素, Dissertation, Shanghai 2012, S. 25-26).

22 Waley: One Hundred \& Seventy Chinese Poems (Anm. 19), S. 157.

23 Brecht: Werke (Anm. 1), Bd. 11, S. 257.

24 Vgl. dazu auch Reinhold Grimm: Bertolt Brecht und die Weltliteratur, Nürnberg 1961, S. 66; Peter Paul Schwarz: Lyrik und Zeitgeschichte. Brecht, Gedichte über das Exil und 
assoziiert, und selbst wenn man keine autobiographische Lesart unterlegt, würde dennoch durch die Umschreibung auch das Bild des Autors als das eines scharfen Gesellschaftskritikers mitkreiert.

Mit Du Fu und Bai Juyi werden also zwei Dichterfiguren heroisiert, die deutschsprachigen linken Autoren als Prototypen einer engagierten Lyrik dienen bzw. durch eine selektive Lektüre und zahlreiche Eingriffe dazu stilisiert werden. Beide werden als Großmeister der Lyrik gefeiert, ihr Heroismus setzt aber einerseits einen gewissen Kampfgeist und andererseits ein herausragendes ethisches Bewusstsein voraus. Das Bild des Dichterhelden zehrt hier schon eindeutig von anderen Heldenbildern. Noch ausgeprägter wird die Überschneidung der Heroismuskonzepte dann bei dem Mann, der auch für einige deutsche Dichter die Synthese aus Ästhet und Kämpfer symbolisieren sollte: Mao Zedong.

\section{Dichterrevolutionär und sensibler Ästhet? Mao Zedong als Dichterbeld}

Die Vorstellung der chinesischen Dichtung als Dichtung der Sozialkritik ist, wie erwähnt, bereits im früheren 20. Jahrhundert verbreitet, aber sie stützt sich lange vor allem auf Autoren des klassischen China. Während in China vor allem mit der Bewegung des 4. Mai schon seit dem frühen 20. Jahrhundert neue literarische Formen und Inhalte in eine spannungsreiche Wechselbeziehung zur Tradition traten, die chinesische Revolution, der Bürgerkrieg und die militärischen Auseinandersetzungen das ganze Land stark veränderten, blieben selbst diejenigen deutschen Dichter, die sich auf die chinesischen Kollegen als Mitstreiter gegen Unrecht und Armut bezogen, weitestgehend auf die Tang-Dichtung bezogen, wohl auch aufgrund der geringen Zahl in Übersetzungen zugänglicher jüngerer Texte. Ab Mitte des 20. Jahrhunderts änderte sich dies, als Mao Zedong nach dem Sieg der Kommunisten nicht nur verstärkt von der deutschen Linken als politischer Führer bewundert wurde, sondern gerade in seiner Doppelrolle als Revolutionär und Dichter rezipiert wurde. Dass Mao sich als Verfasser von Gedichten nach klassischen Formen hervortat, war im Westen seit Edgar Snows Red Star over China (1937) bekannt. Maos Gedichte waren Teil seiner Selbststilisierung. Einerseits gerierte er sich damit als Literatus im klassischen Sinne, andererseits boten sie Raum für Selbstdarstellungen als Führungsfigur der chinesischen Revolution und der jungen Volksrepublik. ${ }^{25}$ Wenige deutsche Fassungen der ersten bekannten MaoGedichte waren ab den frühen 1950er Jahren zugänglich. So spannte Brecht in ei-

späte Lyrik (Literatur und Geschichte 12), Heidelberg 1978, S. 41. Warum Tatlow davon ausgeht, dass eine zynische Lesart nicht möglich sei, leuchtet mir nicht ein (vgl. Anthony Tatlow: Brechts chinesische Gedichte, Frankfurt am Main 1973, S. 45).

25 Vgl. auch Karl-Heinz Pohl: Mao Zedongs Lyrik. Form als Aussage, in: Albrecht Koschorke / Konstantin Kaminskij (Hg.): Despoten dichten. Sprachkunst und Gewalt, Konstanz 2011, S. 227-247, hier S. 232-233. 
ner erweiterten Fassung seiner Chinesischen Gedichte (1950) mit Gedanken bei einem Flug über die Große Mauer nach Maos 沁园春.雪 (Schnee) den Bogen von der sozialkritischen vergangenen Dichtung zur Revolution der Gegenwart. Für die Entdeckung des Dichterrevolutionärs Mao im deutschsprachigen Raum zeichnete aber vor allem Franz Carl Weiskopf verantwortlich. Der tschechisch-deutsche Schriftsteller, der als überzeugter Kommunist die Kriegsjahre im amerikanischen Exil verbracht hatte, diente von 1950 bis 1952 in der tschechischen Botschaft in Beijing. Er eignete sich nicht nur einige Kenntnisse des Chinesischen an, sondern übertrug mit Hilfe chinesischer Bekannter sowie vorliegender Übersetzungen Gedichte vor allem aus der jüngsten Gegenwart. Die zentrale Heldenfigur seiner Sammlung ist in doppelter Hinsicht Mao Zedong: Mao wird einerseits in anderen Gedichten als Retter der Nation, „unsere Sonne“, 26 apostrophiert. Andererseits kommt Mao selbst als Dichter zu Wort. Die vier damals bekannten, von Weiskopf aufgegriffenen Mao-Gedichte fügen sich zu einer Art lyrischen Autobiographie zusammen, wobei er sich zahlreiche Freiheiten im Umgang mit den Texten erlaubte. Letztendlich schwankte Weiskopf in seinen Versuchen, ein Idealbild des Dichterrevolutionärs zu zeichnen, zwischen dem Porträt eines sensiblen, naturaffinen Melancholikers, das sich aus älteren China-Klischees speist, und dem eines stählernen Kommunisten. So wird das Gedicht 沁园春·长沙 (Changsha) von 1925, ${ }^{27}$ das die Anfänge revolutionärer Bande gegen den Hintergrund einer düsteren, gefahrvollen Gegenwart beschwört, bei Weiskopf zu einem Jugendgedicht, in dem ein lyrisches Ich sich seiner rebellischen Jugendzeit erinnert und den Kontrast zwischen Jung und Alt im sentimentalen Wechsel zwischen Präteritum und Präsens beschwört:

$[\ldots]$

Einst schlenderten wir hier, Gefährte mit Gefährt.

Wir hielten uns vertraulich an der Hand.

Wie klar ist mir noch jedes Jahr im Sinn!

Wir waren Schüler, außer Rand und Band

Vor Übermut der Jugend. Alles war

In uns noch Knospe: Herz, Talent, Verstand.

Wir dachten nicht an Ruhm, an Laufbahn und Erfolg.

Die Berge, Flüsse, zeigten wir uns wie im Rausch.

Und trunken war das Wort. Nichts galt

Uns Glanz, nichts Macht der Herrschenden.

Sie waren Moder, Kot; sie waren alt!

Ach, weißt du's noch?

Wie sich die Welle stürmisch hob

Am Bug des Boots im stärksten Strom... ${ }^{28}$

26 F. C. [Franz Carl] Weiskopf: Gesammelte Werke, Bd. 5, Berlin 1960, S. 192-193.

27 Möglicherweise auch 1926, vgl. Jeremy Ingalls: Dragon in Ambush. The Art of War in the Poems of Mao Zedong, Lanham u. a. 2013, S. 135.

28 Weiskopf: Werke (Anm. 26), Bd. 5, S. 271. 
Ein melancholischer Grundton kennzeichnet den Text. Das erinnerte rauschhafte Erleben der Jugend ist dabei ein sinnesbezogenes Erleben der Natur als Gemeinschaftserfahrung im kleinsten Kreis. Im Original ist von „hunderten von Gefährten“ (百侣) die Rede, die die Herrschenden verachten, als Kot ansehen und der Kritik unterziehen. ${ }^{29}$ Wenn auch bei Mao ein gewisser melancholischer Grundton des jetzt einsam im kalten Herbst (独立寒秋) ${ }^{30}$ auf die Landschaft Blickenden zu spüren ist, weist doch die Erinnerung an die früheste Gemeinschaft auf den fortdauernden Kampf hin und enthält einen appellativen Gestus. Das Originalgedicht verzichtet, wie in der klassischen Dichtungssprache häufig, auf jegliche explizite Pronominalverwendung. Bei Weiskopf dagegen scheint der Kontrast zwischen dem reflektierenden Ich der Gegenwart und dem vertraulich verbundenen, mehrfach beschworenen ,Wir auf einen uneinholbaren Gemeinschaftsverlust hinzuweisen. Weiskopfs Interpretation läuft damit der Selbstinszenierung Mao Zedongs in vieler Hinsicht entgegen. An die Stelle des Selbstporträts eines einsamen Revolutionärs, der seiner Anfänge und seiner verstreuten Kameraden gedenkt und daraus Kraft schöpft, aber zugleich von Trauer erfüllt wird, tritt die resignierende Erinnerung eines älter gewordenen Mannes an seine Jugendjahre, wobei das Ich der Vergangenheit einer Art kontextlosen, zeit- und kulturübergreifenden Typus des kraftsprühenden, rebellischen Jungspunds entspricht.

Im Gegensatz dazu ist Weiskopf dann in dem Gedicht Südwörts bemüht, ein ganz explizites Bild eines kommunistischen Kämpfers zu zeichnen:

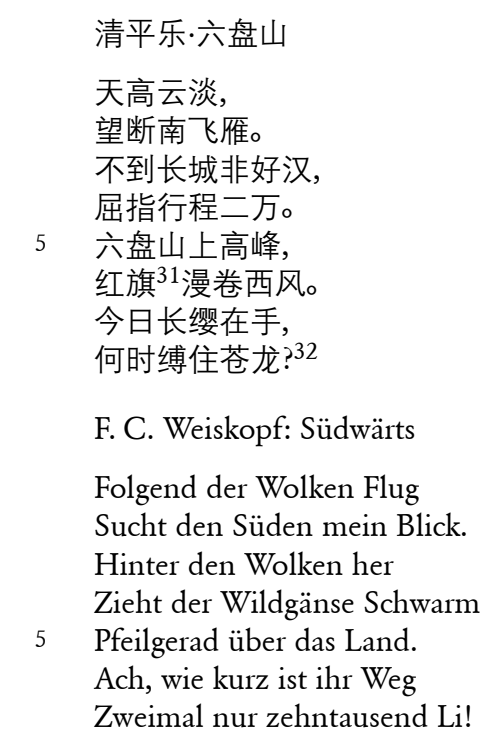

29 Mao Zedong 毛泽东: Gesammelte Lyrik Mao Zedongs 毛泽东诗词集, Beijing 1990, S. 6.

30 Ebd.

31 In der offiziellen Veröffentlichung von 1957 steht 族头 („Banner“), in der Ausgabe von 1963 红旗 (,rote Flagge“), vgl. ebd., S. 66.

32 Ebd., S. 65. 
Und der unsrige, ach, Wieviel mal länger doch!

10 Da nun sage ich mir:

Auch für uns wird es Zeit,

Südwärts zu richten den Schritt.

Kampf ist der Schlüssel zum Weg,

Der uns nach Süden führt,

15 Bitterer Kampf. Es hält

Meine Hand schon umspannt

Fest das Gewehr. Es fragt

Mich mein eigenes Herz:

Wann gelingt uns der Schlag,

20 Der letzte, entscheidende, der

Des Feindes Horden zersprengt?

$\mathrm{Da}$ - wie Antwort darauf,

Stumm und doch laut erblüht

Unsere Fahne rot

25 Auf dem felsigen Hang

Mir gegenüber. Es blühn

Andere hinter ihr auf, zahllose

Wie der Unseren nimmer zu zählende Scharen:

Welle um Welle von hier

30 Bis an den Horizont.

Ja, jetzt ich ich's,

Jetzt weiß es mein Herz: wir werden

Siegreich nach Süden ziehn,

Unaufhaltsam, den Wogen gleich

35 Des gewaltigen Meers.

Und im Wind über uns,

Rot im Wind über uns

Werden die Fahnen wehn,

Die einst Lenin gepflanzt. ${ }^{33}$

Auf den allerersten Blick ist offensichtlich, dass die deutsche Version um ein Vielfaches länger ist als die chinesische. Maos $C i,{ }^{34}$ entstanden kurz vor Ende des langen Marsches, setzt ein mit einer Naturbeobachtung vom Berg in Richtung Himmel und Ferne, zum Zielpunkt der langen Mauer, deren Erreichen erst den Status als

33 Weiskopf: Werke (Anm. 26), Bd. 5, S. 273-274.

$34 \mathrm{Ci}$ bezeichnet eine Gattung, die vor allem in der Song-Zeit populär war. Dabei werden Texte zu überlieferten Melodien bzw. nach diesen rhythmischen Mustern geschrieben. Es handelt sich also um eine durchaus traditionelle, strenge Form, wenn auch mit etwas mehr Freiheiten als bei der shi-Dichtung, bei der die Verse aus fünf oder sieben Silben bestehen und bestimmten Tonmustern folgen. Zur Rolle Maos als Verfasser von Lyrik nach klassischen Formvorgaben vgl. C. N. Tay: Two Poems of Mao Zedong in the Light of Chinese Literary Tradition, in: The Journal of Asian Studies 29.3, 1970, S. 633-655; Haosheng Yang: A Modernity Set to a Pre-Modern Tune. Classical-Style Poetry of Modern Chinese Writers (Ideas, History, and Modern China 14), Leiden/Boston 2016, S. 1-31, S. 147-182; Pohl: Mao Zedongs Lyrik (Anm. 25). 
Held (好汉) rechtfertigen wird. Auf dem Gipfel des Liupan-Bergs wehen aber schon die Banner im Wind, was die Frage aufwirft, wann es gelingen wird, den „dunkelgrünen Drachen/Feind“ (苍龙), sprich Chiang Kai-shek und seine Truppen, zu binden. Ein Ich tritt im Original, wie zumeist in klassischer Dichtung, nicht explizit in Erscheinung. Ohne Pronomen lässt sich die Heroisierung als die des Kollektivs wie auch des Sprechers denken.

F. C. Weiskopfs Version rückt das lyrische Ich dagegen ganz zentral in den Vordergrund und gestaltet das Gedicht als eine Art Zwiegespräch des Ich mit sich selbst, das aus dem Leid und der Entbehrung heraus den Entschluss zum fortgeführten Kampf zieht und schließlich auch, im Bewusstsein der Lenin-Nachfolge, zur Siegesgewissheit gelangt. Der Blick wird damit gelenkt auf die Gefühlslage und den sich sukzessive steigernden Heldenmut des Ich, das die Erbschaft der großen kommunistischen Führer der Vergangenheit antritt und sich selbst auch als Teil dieser Reihe reflektiert. Kampf und lyrische Selbstreflexion sind hier untrennbar miteinander verschränkt. Das Original bietet so nur den Ausgangspunkt zur heroischen Stilisierung des Dichterrevolutionärs Mao nach der Fantasie des deutschen Kommunisten.

Bei aller Mao-Begeisterung läuft Weiskopfs Mao-Stilisierung der Selbstheroisierung des chinesischen Staatsmannes durchaus zuwider und bleibt ambivalent: Dichtertum und Kampf stehen in einem wechselnden Verhältnis zueinander, Dichtung schließt hier naturempfindsames, ungerichtetes Rebellentum ebenso ein wie die Reflexion des eigenen Kampfesmuts.

\section{Möglichkeiten und Grenzen dichterischen Kampfes: Lu Xun}

Ein weiterer chinesischer Dichterheld, dessen Leben eng mit den revolutionären Geschehnissen in China verbunden war, wenn auch sehr viel spannungsreicher, zog die deutschen Schriftsteller in seinen Bann: Lu Xun (鲁迅, auch Lu-Hsün). Der ,Vater der chinesischen Moderne', einer der wichtigsten Vorkämpfer für eine neue, mit Umgangssprache arbeitende Literatur, aber auch ein Erneuerer der Tradition, ${ }^{35}$ ein scharfer Kritiker der Guomindang, der zugleich nie der Kommunistischen Partei beitrat, dürfte Lu Xun wohl vor allem aufgrund der seinem Denken und Schreiben inhärenten Spannungen die deutschen Dichter zur Auseinandersetzung angeregt haben. Nachdem er lange Zeit vor allem in der Bundesrepublik kaum bekannt gewesen war, wurde ihm um 1968 neue Aufmerksamkeit zuteil, zunächst einmal durch Hans Magnus Enzensbergers Kursbuch 15 von 1968. Die meistgelesene Nummer der Kulturzeitschrift aus dem Umfeld der Studentenbewegung stellte die Möglichkeit revolutionärer Dichtung radikal in Frage und löste damit eine Krise unter den linksgerichteten Schriftstellern der Studentenbewegung

$35 \mathrm{Zu} \mathrm{Lu}$ Xuns Rolle als Verfechter einer baihua-Dichtung, einer an die Umgangssprache angenäherten Literatursprache, und als Dichter, der intensiv aus dem klassischen Erbe zehrte und dessen Formen weiterentwickelte, vgl. auch Yang: A Modernity (Anm. 34), S. 32-61. 
vielleicht weniger aus, als dass es sie schon kritisch hinterfragte und ironisierte. In genau dieser Ausgabe wird auch Lu Xun aufgegriffen, einerseits im Porträt eines deutschen Sinologen als dichterischer Vorkämpfer der Revolution, ${ }^{36}$ andererseits in einigen seiner eigenen Essays als Skeptiker ,revolutionärer ${ }^{6}$ Dichtung. ${ }^{37}$ So ist es auch nicht verwunderlich, dass die deutschen Autoren, die Lu Xun durch das Kursbuch entdeckten, ohnehin ein zwiespältiges Verhältnis zum politischen Schreiben hatten. In der Lyrik erwählte sich vor allem Jürgen Theobaldy, der wohl vehementeste Verteidiger der ,Neuen Subjektivität ${ }^{6}{ }^{38}$ Lu Xun zum Bundesgenossen. Theobaldy rückt Lu Xuns Poetologie in die Nähe des eigenen Ansatzes, wobei er sich beinahe ausschließlich auf die oft privateren Gedichte nach klassischen Formen bezieht. Lu Xuns Werk zeichne sich aus durch „Erkenntnis-, Leidens- und Bündnisfähigkeit“ ${ }^{39}$ durch ein Bewusstsein für größere politische Zusammenhänge, eine persönliche Betroffenheit, aber auch gelegentlich den Wunsch nach Rückzug. ${ }^{40}$ Dieses Spannungsverhältnis versucht Theobaldy auch in seinen Lu XunÜbertragungen in Kooperation mit dem Sinologen Egbert Baqué zu gestalten, deren Verfahrensweise exemplarisch an einer Übertragung illustriert werden soll:

无题

惯于长夜过春时, 幋妇将维䯶有丝。 梦里依稀慈母泪, 城头变幻大王旗。

5 忍看朋彗成新鬼, 怒向刀丛受小诗。 吟罢低眉无写处, 月光如水照缁衣。 ${ }^{41}$

Jürgen Theobaldy: Ich erinnere mich, um zu vergessen

Gewohnt bin ich an lange Nächte, der Frühling ist vergangen;

Muß sorgen für die Frau, das Kind, wie Seide grau die Schläfen.

36 Vgl. Joachim Schickel: Dossier: China. Kultur Revolution Literatur, in: Kursbuch 15, 1968, S. 38-62.

37 Lu Hsün: Vier Schriften über Literatur und Revolution, übers. von Rolf Dornbacher / Peter-Anton von Arnim, in: Kursbuch 15, 1968, S. 18-37.

38 Der (umstrittene) Begriff der ,Neuen Subjektivität' bezieht sich auf eine Gruppe junger Autoren, die größtenteils mit politischer Lyrik begonnen hatten und später für das Wiederentdecken des Alltäglichen, gegen das Aussparen des Privaten eintraten, in der Hoffnung, das komplexe Wechselverhältnis zwischen Individuum und Gesellschaft besser greifen zu können. Zur Debatte um diese Lyrikrichtung vgl. v. a. die teils polemischen Beiträge in Akzente 24, 1977.

39 Lu Xun: Kein Ort zum Schreiben. Gesammelte Gedichte, übers. von Egbert Baqué / Jürgen Theobaldy, Reinbek bei Hamburg 1983, S. 116.

40 Vgl. ebd.

41 Ders.: Lu Xuns Gedichte mit Kommentar 鲁迅诗歌注, hg. von Zhou Zhenfu 周振甫, Jiangsu 2006, S. 69. 
5 Verschwommen steht im Traum die Mutter,

in Tränen aufgegangen;

Am Stadtwall wechseln immer wieder

die Herrschenden die Fahnen.

Muß dulden, daß sie meine Freunde

10 ins Reich der Toten treiben;

Steh zornig vor dem Wald der Messer

und suche doch nach Versen.

Ich murmle sie gesenkten Blickes,

hab keinen Ort zum Schreiben;

15 Das Mondlicht fließt, ein helles Wasser

auf meinen schwarzen Kleidern. ${ }^{42}$

Das stark autobiographisch ausgerichtete Gedicht ist 1931 entstanden, nachdem eine Gruppe junger kommunistischer Schriftsteller, unter anderem der mit Lu Xun befreundete Rou Shi (柔石), von der Guomindang hingerichtet worden waren und Lu Xun sich selbst akut bedroht sah. ${ }^{43}$

Theobaldy bemüht sich um eine Annäherung an den Originaltext auf verschiedenen Ebenen, durch Beibehaltung der Bilder und durch eine Tendenz zur subjektlosen Ellipse (durchbrochen nur in V. 13) ebenso wie eine gewisse Strenge der Form in den siebenhebigen, teils gereimten, teils assonierenden Verspaaren, die die Formstrenge des originalen achtversigen Gedichts mit sieben Zeichen pro Zeile vermitteln soll. Auffällig ist jedoch ein Eingriff, der gerade die poetologische Ebene des Gedichts betrifft: So thematisiert der Text das Dichten und den Dichter selbst vor dem Hintergrund der Gewalt und existenziellen Bedrohung. Der sechste Vers, 怒向刀丛受小诗 (wörtl. „zornig Richtung Messerwald suchen kleines Gedicht"), thematisiert das Anschreiben gegen die Gewalt, wobei hier wohl trotziger Kampfeswille und Zweifel an der Wirksamkeit des Schreibens zusammenspielen. Theobaldy schreibt den Vers etwas um: Das Dichter-Ich steht vor dem Wald der Messer, das Gedicht ist nicht mehr explizit gegen diesen gerichtet. Zudem fügt Theobaldy die Adverbiale ,doch` ein. Der Vers wird auf verschiedene Weisen lesbar: Entweder liest man ihn primär als trotzige Selbstbehauptung des Dichtens gegen die Gewalt, ohne dass das Gedicht notwendigerweise selbst ein Kampfgedicht sein muss, es mag wohl auch die Trauer des Ichs reflektieren. Der Widerstand gegen die Gewalt ergibt sich also aus dem Akt des Dichtens an sich. Andererseits ließe sich der Vers deuten als skeptische Hinterfragung des Dichtens in Reaktion auf Mord und Bedrohung, dann wiese das ,doch ${ }^{6}$ eher darauf hin, dass sich das Ich trotz seines Zornes zur ästhetischen Bearbeitung hinreißen lässt, anstatt sich mit anderen Mitteln den Mächtigen entgegenzustellen. Dichten ist demnach einerseits per se Widerstand gegen die Macht, indem es nicht unterdrückt werden kann, andererseits ist es auch eine gewisse eskapistische Geste, die ambivalent bleibt. Der Dichter bleibt so unweigerlich

42 Lu Xun: Kein Ort (Anm. 39), S. 43.

43 Vgl. ders.: Gedichte mit Kommentar (Anm. 41), S. 69. 
dem politischen Geschehen der Zeit verbunden, von ihm betroffen, aber nimmt dennoch eine andere Position ein als ein Kämpfer. Dichter- und Kampfesheld driften hier also mindestens partiell wieder auseinander.

\section{Zusammenfassung: Der Held als Ästhet, Ästhetik des Heldentums}

Zusammenfassend sei festzuhalten: Zahlreiche deutsche Dichter des letzten Jahrhunderts haben sich für die chinesische Dichtung und die chinesischen Dichter interessiert. Gerade in Krisen- und Umbruchszeiten nahmen sie auf sie Bezug, um eigene Ansätze zu rechtfertigen und sich über die Rolle des Dichters zu verständigen. Damit erstrebten sie eine Art zeit- und kulturübergreifende Legitimation der eigenen Position, wobei das Bild der chinesischen Dichter wiederum selbst bis zum gewissen Grad ein Produkt der projektiven Überformung seitens der deutschen Dichter ist. Diese schrieben Gedichte über die Dichter, sie gestalteten Paratexte, die vor allem auf die Person der Dichter fokussiert sind, und sie schrieben die Gedichte der chinesischen Kollegen selbst auf eine Art und Weise fort, die oft einen Dialog mit der eigenen Dichtung erkennen lässt. Nicht zuletzt aufgrund autobiographischer Suggestionen scheint ein bestimmtes Dichter-Idealbild durch. Die chinesischen Dichterhelden werden in dieser Indienstnahme auf spezifische Dichter-Typen reduziert, auf das melancholische, trunkene und naturverbundene Genie, den Kämpfer für Gerechtigkeit bis hin zum Dichterrevolutionär oder aber den zwischen Revolution und Kontemplation hin- und hergerissenen Dichter. Das Konzept des Dichterhelden steht in einer spannungsvollen Beziehung zu anderen Vorstellungen des Heroischen. Der Dichter kann sich als Rebell gesellschaftlichen Konventionen und damit auch den Ansprüchen des Heldentums versagen und damit eher zum Antiheld werden. Er kann in der Stilisierung als Kämpfer für Gerechtigkeit an andere Heroentypen angelehnt werden oder aber bei aller Engagiertheit einen gewissen autonomen Status für sich beanspruchen. Anhand der Auslotung der Heldenkonzepte verhandelten die deutschen Dichter somit letztlich auch ihre eigene Position in Gesellschaft und Politik. 